\title{
Persistent photoconductivity and carrier transport in AIGaN/GaN heterostructures treated by fluorine plasma
}

\author{
B. K. Li, ${ }^{1}$ W. K. Ge, ${ }^{1}$ J. N. Wang, ${ }^{1, a)}$ and K. J. Chen ${ }^{2}$ \\ ${ }^{1}$ Department of Physics, The Hong Kong University of Science and Technology, Clear Water Bay, Kowloon, \\ Hong Kong, China \\ ${ }^{2}$ Department of Electronic and Computer Engineering, The Hong Kong University of Science \\ and Technology, Clear Water Bay, Kowloon, Hong Kong, China
}

(Received 30 December 2007; accepted 5 February 2008; published online 27 February 2008)

\begin{abstract}
Fluorine plasma treatment technique can effectively incorporate fluorine atoms into the AlGaN barrier, depleting the two-dimensional electron gases (2DEGs) in the channel of $\mathrm{AlGaN} / \mathrm{GaN}$ high electron mobility transistors and converting the device from depletion mode to enhancement mode. To reveal the underlying physical mechanisms, temperature dependent persistent photoconductivity (PPC) and Hall measurements are conducted in $\mathrm{AlGaN} / \mathrm{GaN}$ heterostructures treated by $\mathrm{CF}_{4}$ plasma. Weakly temperature dependent 2DEG mobility and much more pronounced PPC effect are observed in the F-treated sample. An energy barrier of $624 \mathrm{meV}$ for electrons recaptured by the F-related centers is extracted from the PPC decay behaviors. () 2008 American Institute of Physics.
\end{abstract} [DOI: $10.1063 / 1.2888743$ ]

Wide-bandgap AlGaN/GaN high electron mobility transistors (HEMTs) exhibit inherent advantages for high-power, high-voltage, and high-temperature applications. Performance of the conventional depletion-mode (D-mode) HEMTs (with a negative threshold voltage) has been significantly improved, ${ }^{1}$ while the implementation of enhancementmode (E-mode) or normally off devices (with a positive threshold voltage) is still hindered by technical difficulties. From the application viewpoint, E-mode HEMTs are strongly required because of the circuit simplicity brought by elimination of the negative supply voltage. For digital applications, the simplest circuit configuration can be achieved by using direct-coupled field-effect transistor logic that features integration of D-mode and E-mode HEMTs. For highvoltage power switches, normally off operation is mandatory for device safety. Recently, a robust technique for fabricating E-mode $\mathrm{AlGaN} / \mathrm{GaN}$ HEMTs with low on resistance and low knee voltage starting from a conventional D-mode HEMT structure has been reported. ${ }^{2,3}$ The technique is based on self-aligned fluorine plasma treatment and post-gateannealing. It has been found that the fluorine ions are responsible for the conversion from $\mathrm{D}$ mode to $\mathrm{E}$ mode. The question of how F ions act in the III-nitride material system is of fundamental importance to further understand and improve the fluorine plasma treatment technique. A fluorine atom possesses strong electronegativity, but virtually no knowledge exists about its behavior as an impurity in III-nitride semiconductors. In this work, we report a study of F-related centers in an $\mathrm{AlGaN} / \mathrm{GaN}$ heterostructure using temperature dependent Hall effect and persistent photoconductivity (PPC) measurements.

The $\mathrm{AlGaN} / \mathrm{GaN}$ epitaxial structure used in this work was a base line $\mathrm{AlGaN} / \mathrm{GaN}$ HEMT structure grown on (0001) sapphire substrate in a metal organic chemical vapor deposition system. The epitaxial structure consists of a lowtemperature $\mathrm{GaN}$ nucleation layer, a 2.5 - $\mu \mathrm{m}$-thick unintentionally doped $\mathrm{GaN}$ buffer layer, and an $\mathrm{AlGaN}$ barrier layer

${ }^{a)}$ Electronic mail: phjwang@ust.hk. with 30\% Al composition. The barrier layer consists of a $3 \mathrm{~nm}$ undoped spacer, a $15 \mathrm{~nm}$ carrier supplier layer $\mathrm{Si}$ doped at $2.5 \times 10^{18} \mathrm{~cm}^{-3}$, and a $2 \mathrm{~nm}$ undoped cap layer. The Ohmic contacts were formed using $\mathrm{Ti} / \mathrm{Al} / \mathrm{Ni} / \mathrm{Au}$ metallization with a contact resistance of $0.8 \Omega \mathrm{mm}$. The sample for Hall effect and PPC measurements was treated with $\mathrm{CF}_{4}$ plasma in a reactive ion etching system at a rf power of $150 \mathrm{~W}$ for $120 \mathrm{~s}$, followed by a rapid thermal annealing at $400{ }^{\circ} \mathrm{C}$ for $10 \mathrm{~min}$. The post-treatment annealing has been shown to be effective in repairing the plasma-induced damage and the annealing temperature is low enough to be compatible with the gate process. Another sample without the fluorine treatment was also prepared and measured for comparison. Temperature dependent (80-305 K) Hall measurements were performed using the van der Pauw configuration. An Ar-ion laser was used for PPC measurements. A bias of $200 \mathrm{mV}$ was applied between two contacts with $\sim 150 \mu \mathrm{m}$ spacing. The data obtained under different conditions were taken in such a way that the sample was always allowed to relax to equilibrium.

The electron density and mobility of both the treated and untreated samples are measured in the dark with temperature varying from 80 to $305 \mathrm{~K}$, as shown in Fig. 1. Both the carrier density and mobility of the untreated sample show typical temperature dependence of two-dimensional electron gas (2DEG). ${ }^{4}$ while for the F-plasma treated sample, both the carrier density and mobility decrease drastically. It is well known that fluorine is the most electronegative element and the $\mathrm{CF}_{4}$ plasma generates a great deal of negatively charged fluorine ions. It is reasonable to assume that the $\mathrm{F}$ ions are negatively charged in the heterostructure when most of them are concentrated in the AlGaN barrier, as shown by the secondary ion mass spectroscopy (SIMS). ${ }^{3}$ These $\mathrm{F}^{-}$ions can thus deplete the 2DEG in the channel and decrease the carrier density, leading to a positive shift in threshold voltage. While the decrease of carrier mobility after the F-plasma treatment mainly results from reduced carrier density and increased impurity scattering. The decrease of carrier density indicates that both the quantum confinement for $2 \mathrm{DEG}$ at the 


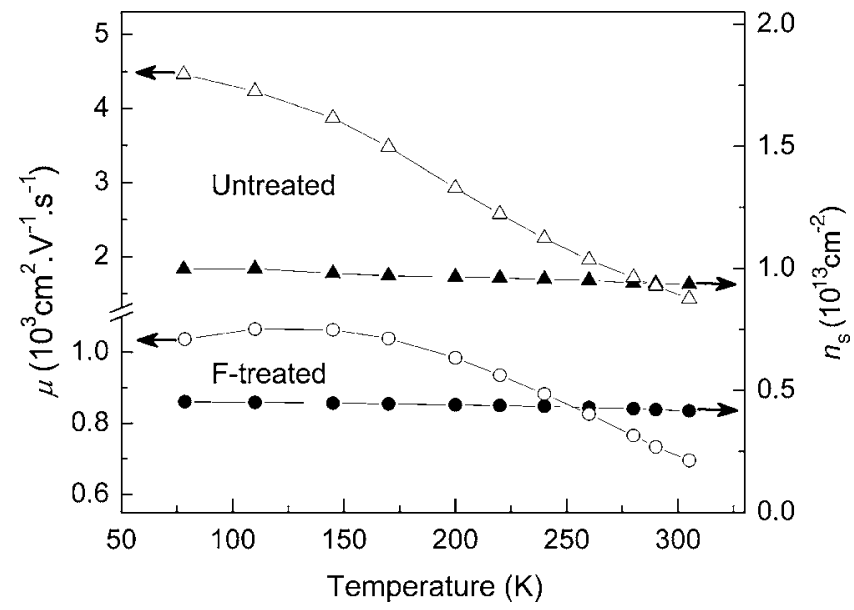

FIG. 1. 2DEG carrier density (solid) $n_{s}$ and mobility (open) $\mu$ of the F-plasma treated (circle) and untreated (triangle) $\mathrm{AlGaN} / \mathrm{GaN}$ heterostructures as a function of temperature $T$, measured in the dark.

interface of $\mathrm{AlGaN} / \mathrm{GaN}$ heterostructure and the screening effect are weakened. In addition, the depth profile of the fluorine atoms measured by SIMS (Ref. 3) shows that a small amount of fluorine atoms can penetrate the AlGaN barrier and be presented into the GaN channel. These fluorine atoms, which are negatively charged, increase the carrier scattering rate. Another interesting phenomenon is that the electron mobility in the treated sample reaches a maximum at about $110 \mathrm{~K}$. This temperature dependence of the mobility in the treated sample is similar to that in bulk GaN epilayers, where the impurity scattering dominates, ${ }^{5,6}$ suggesting that impurity scattering (by fluorine atoms) in the 2DEG channel plays a dominating role in limiting the 2DEG mobility in the F-treated sample at low temperature. This result is consistent with the fact that a small amount of fluorine ions end up in the channel, as observed from the fluorine ion distribution profiles. $^{3}$

The phenomenon of the PPC effect has been observed in $\mathrm{GaN}$ thin films ${ }^{7,8}$ and $\mathrm{AlGaN} / \mathrm{GaN}$ heterostructures. ${ }^{4,9}$ The investigation of the PPC effect in these materials can provide information on the deep center levels and the carrier relaxation. Here, we report the PPC effect observed in $\mathrm{AlGaN} / \mathrm{GaN}$ heterostructures treated by fluorine plasma. An Ar-ion laser $(\lambda=488 \mathrm{~nm})$ was used as the excitation source. Figure 2 shows the PPC decay curves of the treated and untreated samples after excitation using a $488 \mathrm{~nm}$ light under the same conditions at room temperature. $I_{\mathrm{PC}}$ is defined as the difference between the measured photocurrent and the dark current $\left(I_{\text {dark }}\right)$. The treated sample shows a much more pronounced PPC effect than the untreated sample. The decay time constant of the treated sample is much larger than that of the untreated sample. The large decay time constant allows us to investigate the dependence of the electron mobility on the carrier density of the treated sample by conducting Hall measurement at various times after the light excitation was turned off. The inset of Fig. 2 shows the results obtained at room temperature, indicating that the mobility increases with the increase of sheet density of the 2DEG. This behavior is different from that for $\mathrm{GaN}$ epilayers ${ }^{6}$ but similar to that for the $2 \mathrm{DEG}$ in $\mathrm{AlGaAs} / \mathrm{GaAs}$ (Ref. 10) and $\mathrm{AlGaN} / \mathrm{GaN}^{4}{ }^{4}$ The main reason for the improved electron mobility at higher 2DEG concentration is the reduced impu-

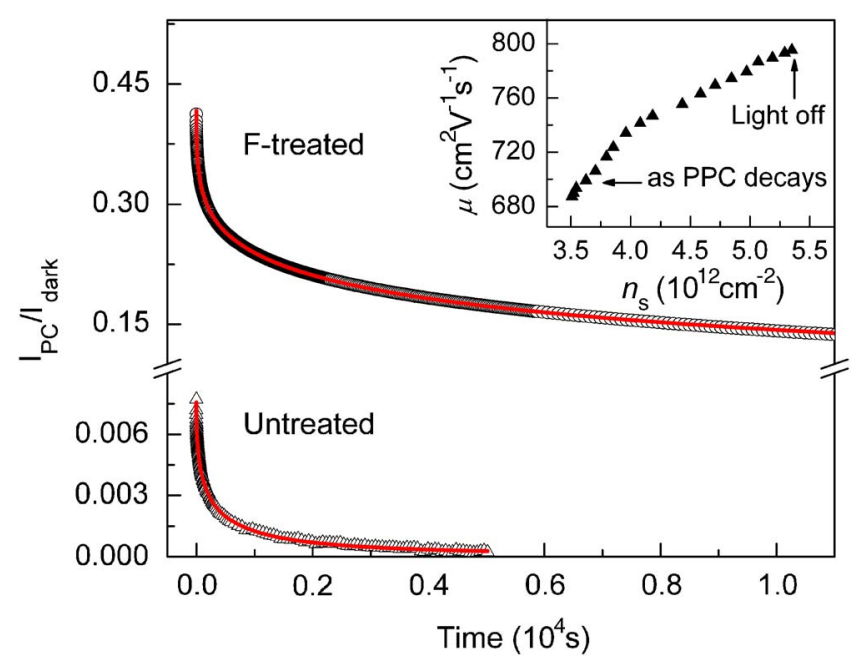

FIG. 2. (Color online) The PPC decay behavior of the treated and untreated samples at room temperature. The samples were excited by a $488 \mathrm{~nm}$ Ar-ion laser source with the same intensity and to a saturation state. The solid curves are the stretched-exponential fit to the data. The inset shows the 2DEG carrier mobility $\mu$ as a function of carrier density $n_{s}$ of the treated $\mathrm{AlGaN} / \mathrm{GaN}$ heterostructure, measured at room temperature.

rity and lattice (or phonon) scattering by stronger screening effect.

The decay of the PPC follows a stretched-exponential function, ${ }^{4,9}$

$$
I_{\mathrm{PPC}}(t)=I_{\mathrm{PPC}}(0) \exp \left[-(t / \tau)^{\beta}\right], \quad 0<\beta<1,
$$

where $I_{\mathrm{PPC}}(0)$ is the PPC buildup level at the moment of light excitation being switched off, $\tau$ is the PPC decay time constant, and $\beta$ is the decay exponent. The solid lines in Fig. 2 are the least squares fit of data with Eq. (1). We have measured the PPC behavior of the treated sample at different temperatures and obtained the decay time constants and decay exponents by fitting the decay curves using Eq. (1). Figure 3 shows the Arrhenius plot of the fitted time constants of the treated sample. At temperatures $T>200 \mathrm{~K}, \tau$ exhibits thermally activated behavior. An energy barrier $E_{\mathrm{rc}}$ for the recapture of electrons by the deep F-related centers is esti-

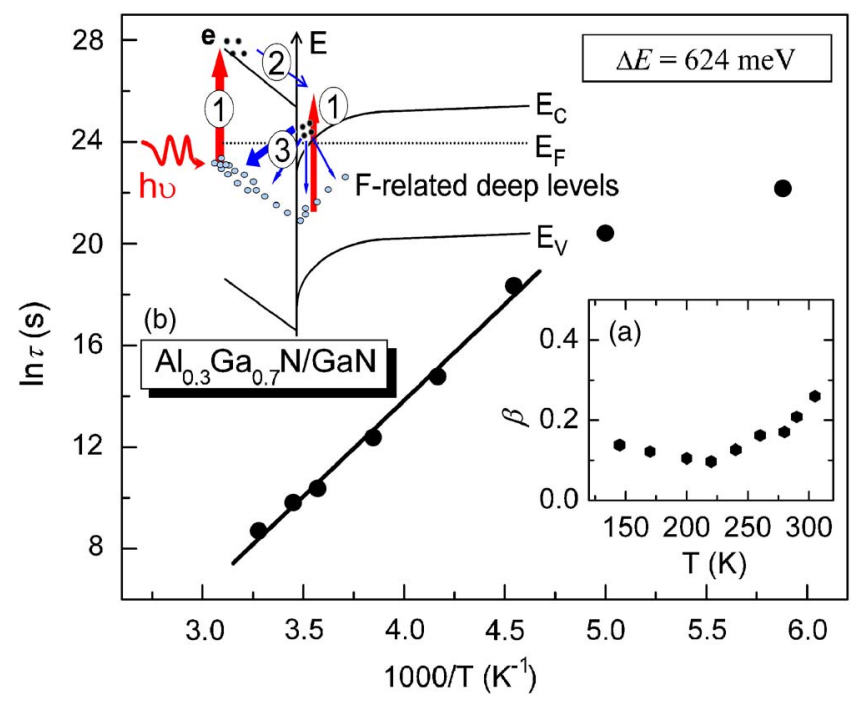

FIG. 3. (Color online) Arrhenius plot of the PPC decay time constant $\tau(\ln \tau$ vs $1000 / T$ ). Inset (a) shows the decay exponent $\beta$ as a function of temperature. Inset (b) illustrates the photoexcitation, drift, and relaxation processes of electrons in the fluorine plasma treated sample.

to AIP license or copyright; see http://apl.aip.org/apl/copyright.jsp 


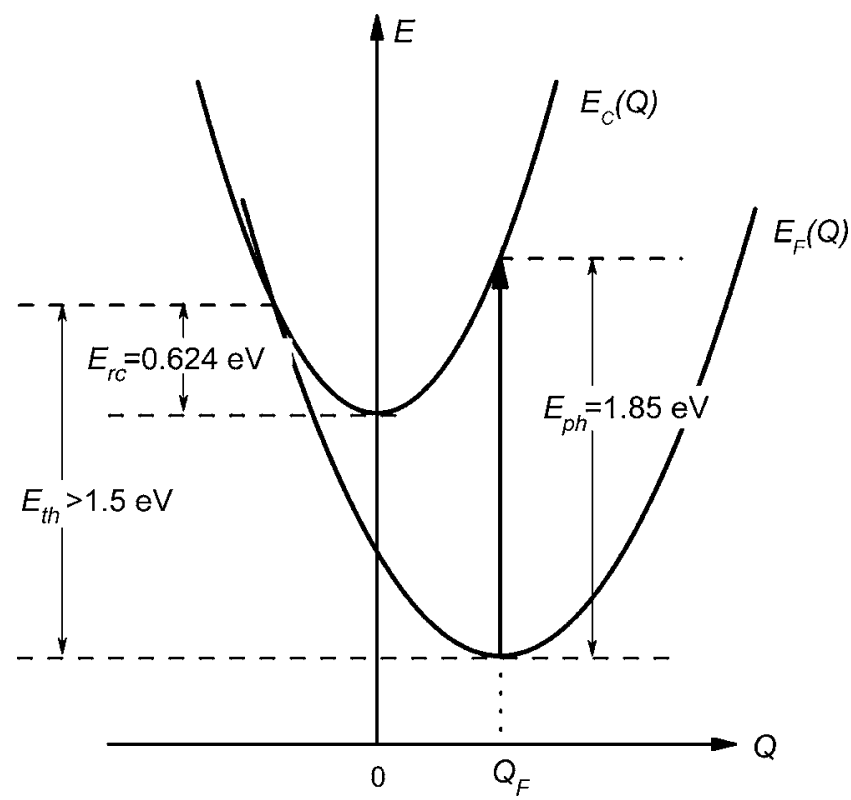

FIG. 4. Configuration coordinate diagram of the F-related deep centers in the $\mathrm{AlGaN} / \mathrm{GaN}$ heterostructure. $E_{C}(Q)$ and $E_{F}(Q)$ are the sums of the electronic and elastic energies corresponding to the conduction band and to the occupied F-related deep centers, respectively, as a function of configuration coordinate $Q . E_{\mathrm{ph}}, E_{\mathrm{th}}$, and $E_{\mathrm{rc}}$ are the photoionization energy, thermal activation energy, and electron recapture energy barrier of the F-related deep centers, respectively.

mated as $624 \mathrm{meV}$. The fitted values of the PPC decay exponent $\beta$ are shown in inset (a) of Fig. 3. The observed PPC effect is illustrated in inset (b) of Fig. 3. Upon light illumination, the electrons trapped by F-related centers are excited (process 1 in the figure); then the photoexcited electrons drift to the channel region (process 2 in the figure), increasing the 2DEG density and contributing to the observed photoconductivity. When the light illumination is off, photoexcited electrons will be recaptured slowly by these deep centers (process 3 in the figure). As the F-related centers are mainly located at the top region of the AlGaN capping layer, ${ }^{3}$ there is a large spatial separation between the photoexcited electrons in the channel and F-related centers. As a result, we observe a larger recapture energy barrier $E_{\mathrm{rc}}$ and a smaller decay exponent $\beta$ compared with those reported for other PPC effects in conventional $\mathrm{AlGaN} / \mathrm{GaN}$ heterostructures ${ }^{4}$ and GaN films. ${ }^{8}$ Although the physical significance of $\beta$ is not clear, the temperature dependence of $\beta$ implies that the decay mechanism at low temperatures is different from that at high temperatures.

Photocurrent spectra of both the treated and untreated samples have been measured at room temperature. ${ }^{11}$ By comparing the photocurrent spectra of the treated and untreated samples measured at the same conditions, the photoioniza- tion energy $E_{\mathrm{ph}}$ of the F-related deep centers in the $\mathrm{AlGaN} / \mathrm{GaN}$ heterostructure was obtained to be $1.85 \mathrm{eV}$, at which the photocurrent of the treated sample started to exhibit a much higher increasing rate compared to the untreated sample. AlGaN/GaN Schottky barrier diodes (SBDs) with and without fluorine plasma treatment, have been characterized using deep-level transient spectroscopy (DLTS). ${ }^{12} \mathrm{~A}$ dominant high-temperature trap, which is associated with the F-related deep centers, is observed for the treated SBD, and the thermal activation energy $E_{\text {th }}$ is estimated to be larger than $1.5 \mathrm{eV}$. On the basis of the experimental results of the temperature dependent PPC, photocurrent spectra, and DLTS measurements, we propose a simple configuration coordinate diagram for the F-related deep centers in the $\mathrm{AlGaN} / \mathrm{GaN}$ heterostructure, as shown in Fig. 4.

In conclusion, we have studied the F-plasma treated $\mathrm{AlGaN} / \mathrm{GaN}$ heterostructure using temperature dependent Hall effect and PPC measurements. Both the 2DEG carrier density and mobility decreased drastically after the F-plasma treatment and a much more pronounced PPC effect is observed in the treated sample, indicating that F-related centers are deep electron traps. The energy barrier for the 2DEG electrons recaptured by the F-related centers is obtained to be $624 \mathrm{meV}$.

This work was supported by the Research Grant Council of Hong Kong SAR government via Grant Nos. SBI07/ 08.SC08, 603704, and 611706. The authors are grateful to Professor Kei May Lau for providing the epitaxial sample for this work.

${ }^{1}$ J. W. Johnson, E. L. Piner, A. Vescan, R. Therrien, P. Rajagopal, J. C. Roberts, J. D. Brown, S. Singhal, and K. J. Linthicum, IEEE Electron Device Lett. 25, 459 (2004).

${ }^{2}$ Y. Cai, Y. G. Zhou, K. J. Chen, and K. M. Lau, IEEE Electron Device Lett. 26, 435 (2005).

${ }^{3}$ Y. Cai, Y. G. Zhou, K. M. Lau, and K. J. Chen, IEEE Trans. Electron Devices 53, 2207 (2006).

${ }^{4}$ J. Z. Li, J. Y. Lin, H. X. Jiang, M. Asif Khan, and Q. Chen, J. Appl. Phys. 82, 1227 (1997).

${ }^{5}$ S. Nakamura, T. Mukai, and M. Senoh, J. Appl. Phys. 71, 5543 (1992).

${ }^{6}$ V. W. L. Chin, T. L. Tansley, and T. Osotchan, J. Appl. Phys. 75, 7365 (1994).

${ }^{7}$ C. H. Qiu and J. I. Pankove, Appl. Phys. Lett. 70, 1983 (1997).

${ }^{8}$ H. M. Chen, Y. F. Chen, M. C. Lee, and M. S. Feng, J. Appl. Phys. 82, 899 (1997).

${ }^{9}$ T. Y. Lin, H. M. Chen, M. S. Tsai, Y. F. Chen, F. F. Fang, C. F. Lin, and G. C. Chi, Phys. Rev. B 58, 13793 (1998).

${ }^{10}$ H. L. Störmer, A. C. Gossard, G. Wiegmann, and K. Baldwin, Appl. Phys. Lett. 39, 912 (1981).

${ }^{11}$ B. K. Li, K. J. Chen, K. M. Lau, W. K. Ge, and J. N. Wang, Seventh International Conference on Nitride Semiconductors, Las Vegas, NV, 2007 (unpublished).

${ }^{12}$ L. W. Lu, Y. Cai, L. Ding, W. K. Ge, K. M. Lau, and K. J. Chen, 49th Electronic Materials Conference, Notre Dame, IN, 2007 (unpublished). 